\title{
Periodic orbit theory of strongly anomalous transport
}

\author{
Roberto Artuso $†$ and Giampaolo Cristadoro \\ Center for Nonlinear and Complex Systems and Dipartimento di Scienze Chimiche, \\ Fisiche e Matematiche, Università dell'Insubria and Istituto Nazionale di Fisica della \\ Materia, Unità di Como, Via Valleggio 11, 22100 Como, Italy \\ E-mail: Roberto.Artuso@uninsubria.it, Giampaolo.Cristadoro@uninsubria.it
}

\begin{abstract}
We establish a deterministic technique to investigate transport moments of arbitrary order. The theory is applied to the analysis of different kinds of intermittent one-dimensional maps and the Lorentz gas with infinite horizon: the typical appearance of phase transitions in the spectrum of transport exponents is explained.

PACS numbers: 05.45.-a
\end{abstract}




\section{Introduction}

It is well known that chaotic dynamical systems may exhibit deterministic diffusion: among the simplest examples one may recall chains of 1-d maps [1, area preserving maps on the cylinder 2] and infinite domain billiards, like the Lorentz gas with finite horizon [3]. In many cases the mean square displacement (the average being taken with respect to initial conditions) displays a linear asymptotic growth

$$
\left\langle\left(x_{t}-x_{0}\right)^{2}\right\rangle \sim D t
$$

and one may try to devise techniques to compute the diffusion constant $D$. In particular periodic orbits expansions have been employed [4] to evaluate $D$ for one dimensional maps [5], finite horizon Lorentz gas [6] and area preserving sawtooth maps 7]. Deterministic transport may also exhibit an anomalous character, with a mean square displacement deviating from a linear behaviour. Typically this is associated to a weakening of chaotic properties, like marginal fixed points [8], the presence of regular islands in the chaotic sea [9], or the opening of ballistic channels for Lorentz gas models 10. Such weakly chaotic regimes are generically quite hard to study, as they miss the fast memory loss that typically characterizes strongly chaotic systems. Besides transport properties this lack of full chaos deeply modifies other dynamical features: for instance escape processes may be slowed down and time correlation functions acquire power law tails (in view of Green-Kubo formula [1] this is closely connected to anomalous diffusion). Global properties of the transport process are investigated through the asymptotic behaviour of generalized moments

$$
\left\langle\left|x_{t}-x_{0}\right|^{q}\right\rangle \sim t^{\nu(q)}
$$

Gaussian processes yield $\nu(q)=q / 2$ for all $q$; recently deviations from a constant slope for $\nu(q)$ have been referred to as "strong" anomalous diffusion [12] (the reader may consult this reference for a wide survey of systems falling in this cathegory). In this paper we will introduce a cycle expansions technique for computing the spectrum $\nu(q)(q / 2 \in \mathbb{N})$ and apply the formalism to a number of examples: different chains of one-dimensional intermittent maps, and infinite domain billiards (Lorentz gas with infinite horizon).

The paper is organized as follows: in section 2, to make the paper self contained, we review how periodic orbit expansions may be employed to study transport processes, section 3 briefly explains how full chaos generically results in a gaussian $\nu(q)$, while, finally, section 4 is devoted to the analysis of weakly chaotic systems yielding a non trivial $\nu(q)$ (parts of the results of this section were anticipated in [13]).

\section{Deterministic transport}

Chaotic dynamical systems are characterized by sensitive dependence on initial conditions, so that a meaningful theory must concentrate on statistical properties, rather 
than on analysis of single trajectories. A central role in such a context is played by the Perron-Frobenius operator $\mathcal{L}$ which acts as:

$$
[\mathcal{L} \phi](x)=\int_{X} \mathrm{~d} y \delta(f(y)-x) \phi(y)
$$

(we are considering for simplicity dynamical evolution described by a discrete mapping $f$ ). This operator may be interpreted as the evolution on densities (dual picture with respect to Koopman dynamics on observables)

$$
\rho_{n+1}(x)=\mathcal{L}\left[\rho_{n}\right](x)=\int_{X} \mathrm{~d} y \delta(f(y)-x) \rho_{n}(y)
$$

(in particular the invariant density is an eigenvector of $\mathcal{L}$, with corresponding eigenvalue $\lambda=1$ ). Notice that the kernel is singular, so that investigation of spectral properties generally requires sophisticated techniques [14]: we also observe that this operator enjoys the semigroup property:

$$
\left[\mathcal{L}^{m} \phi\right](x)=\left[\mathcal{L}^{m-k}\left[\mathcal{L}^{k} \phi\right]\right](x)
$$

The study of transport properties is conveniently carried out in terms of the generating function:

$$
G_{t}(\beta)=\left\langle\mathrm{e}^{\beta\left(x_{t}-x_{0}\right)}\right\rangle
$$

when again the average is over a set of initial conditions. We also define a generalized transfer operator where the singular delta kernel in (3) is weighted by an appropriate factor (that maintains the semigroup property):

$$
\left[\mathcal{L}_{\beta} \phi\right](x)=\int_{X} \mathrm{~d} y \mathrm{e}^{\beta(f(y)-y)} \delta(f(y)-x) \phi(y)
$$

The key idea is that if we are able to derive an analytic expression for the generating function, we can then extract all transport moments:

$$
\left\langle\left(x_{t}-x_{0}\right)^{q}\right\rangle=\left.\left(\frac{\partial^{q}}{\partial \beta^{q}} G_{t}(\beta)\right)\right|_{\beta=0}
$$

The generating function may be expressed as

$$
G_{t}(\beta)=\int \mathrm{d} x\left[\mathcal{L}_{\beta}^{t} \rho_{\text {in }}\right](x)
$$

where $\rho_{\text {in }}$ is the density of initial conditions: the asymptotic growth of $G_{t}(\beta)$ in this way is dominated by the leading eigenvalue of the generalized transfer operator, as in equilibrium statistical mechanics of lattice systems, namely

$$
G_{t}(\beta) \sim \lambda^{t}(\beta)
$$

and then

$$
\left.\left\langle\left(x_{t}-x_{0}\right)^{q}\right\rangle \sim\left(\frac{\partial^{q}}{\partial \beta^{q}} \lambda^{t}(\beta)\right)\right|_{\beta=0}
$$

The main problem thus consists in computing the leading eigenvalue (and its derivatives): before turning to this point we have to describe the particular class of 
dynamical systems we want to study: generally we are interested in spatially extended periodic systems where the dynamics over the unbounded phase space is generated by replicas of a map on a finite fundamental domain (like the Lorentz gas on a square lattice, where the dynamics on a fundamental cell corresponds to the Sinai billiard). From now on we illustrate the case of a one-dimensional map on the real line, even though the method is by no means limited to this context. The key property of the dynamical system under investigation is represented by the symmetry properties

$$
\begin{aligned}
& f(-x)=-f(x) \\
& f(x+n)=n+f(x)
\end{aligned}
$$

for any $n \in \mathbb{Z}$. The property (12) guarantees the absence of a net drift while with (13) we impose that the map on the real line is obtained by lifting a circle map $\hat{f}(\theta)$ defined on the unit torus as

$$
\hat{f}(\theta)=\left.f(\theta)\right|_{\bmod 1} \quad \theta \in \mathcal{T}=[0,1)
$$

We may thus split the $f$ evolution into a box integer plus a fractional part: $x_{n}=N_{n}+\theta_{n}$, where

$$
\begin{aligned}
& N_{n+1}=N_{n}+\sigma\left(\theta_{n}\right) \\
& \theta_{n+1}=\hat{f}\left(\theta_{n}\right)
\end{aligned}
$$

where $\sigma(\theta)=[f(\theta)]$ is the function returning the integer part of the argument.

When considering the generating function (6) translation invariance of the system permits to restrict the average over the elementary cell. The transfer operator on the fundamental cell is written as:

$$
\left[\mathcal{L}_{\beta} \rho\right](\phi)=\int_{\mathcal{T}} \mathrm{d} \theta \mathrm{e}^{\beta(\hat{f}(\theta)-\theta+\sigma(\theta))} \delta(\hat{f}(\theta)-\phi) \rho(\theta)
$$

where all quantities refer to the torus map once we attach to every point in $\mathcal{T}$ the correspondent jumping number $\sigma(x)=[f(x)][\underline{5},[6]$.

The leading eigenvalue is the inverse of the smallest $z$ solving the secular equation:

$$
\operatorname{det}\left(1-z \mathcal{L}_{\beta}\right)=F_{\beta}(z)=0
$$

$\left(F_{\beta}(z)\right.$ is usually called the spectral determinant). Using the formal relationship:

$$
\operatorname{det}\left(1-z \mathcal{L}_{\beta}\right)=\exp \operatorname{Tr} \ln \left(1-z \mathcal{L}_{\beta}\right)=\exp \left(-\sum_{n=1}^{\infty} \frac{z^{n}}{n} \operatorname{Tr} \mathcal{L}_{\beta}^{n}\right)
$$

we are lead to computing the formal trace of $\mathcal{L}_{\beta}^{n}$ :

$$
\begin{aligned}
\operatorname{Tr} \mathcal{L}_{\beta}^{n} & =\int \mathrm{d} x \delta\left(\hat{f}^{n}(x)-x\right) \mathrm{e}^{\left(\hat{f}^{n}(x)-x+\sigma_{n}(x)\right)}= \\
& =\sum_{x=\hat{f}^{n}(x)} \frac{\mathrm{e}^{\left(\beta \sigma_{n}(x)\right)}}{\left|1-\Lambda_{n}(x)\right|}
\end{aligned}
$$


where

$$
\begin{aligned}
& \Lambda_{n}(x)=\prod_{i=0}^{n-1} \hat{f}^{\prime}\left(\hat{f}^{i}(x)\right) \\
& \sigma_{n}(x)=\sum_{j=1}^{n} \sigma\left(f^{j}(x)\right)
\end{aligned}
$$

and the sum runs over all periodic orbits of the system under study.

We may now proceed to derive the standard expression for spectral determinants and zeta functions: the first step consists in using a geometric series expansion for the denominator in (21):

$$
\operatorname{Tr} \mathcal{L}_{\beta}^{n}=\sum_{k=0}^{\infty} \sum_{x=\hat{f}^{n}(x)} \frac{\mathrm{e}^{\left(\beta \sigma_{n}(x)\right)}}{\left|\Lambda_{n}(x)\right|\left(\Lambda_{n}(x)\right)^{k}}
$$

We remark that the geometric series expansion makes sense only if we deal with a hyperbolic system, for which $\left|\Lambda_{n}(x)\right|>1$ for any point and iteration order (differently from what happens for intermittent systems). Next we perform a subtle step: the sum is over periodic points of any possible order $n$ : so for instance fixed points appear at any order, and every $p$ orbit reappears whenever $p$ divides $n$. Moreover all points of a given periodic orbit contribute to the trace, but they all yield the same weight, as instabilities and jumping numbers $\sigma_{n}(x)$ are the same for every points of the same periodic orbit. So we may convert the sum over periodic points to a sum over orbits $p$, each taken once: we furher denote by $\Lambda_{p}$ and $\sigma_{p}$ respectively the instabilities and jumping numbers of the orbit $p$, each computed with respect to its prime period $n_{p}$. In this way we get:

$$
\operatorname{det}\left(1-z \mathcal{L}_{\beta}\right)=\exp \left(-\sum_{\{p\}} \sum_{r=1}^{\infty} \sum_{k=0}^{\infty} \frac{z^{r \cdot n_{p}}}{r} \frac{\mathrm{e}^{\beta \sigma_{p} \cdot r}}{\left|\Lambda_{p}\right|^{r} \Lambda_{p}^{k \cdot r}}\right)
$$

where $\{p\}$ denotes the set of periodic orbits, each taken once, $n_{p}$ being the prime period of the orbit. The sum over $r$ reproduces each repetition of a single orbit $p$ in the original summation over periodic points: this sum may be performed exactly, leading to:

$$
\begin{aligned}
\operatorname{det}\left(1-z \mathcal{L}_{\beta}\right) & =\exp \left(\sum_{\{p\}} \sum_{k=0}^{\infty} \ln \left(1-\frac{z^{n_{p}} \mathrm{e}^{\beta \sigma_{p}}}{\left|\Lambda_{p}\right| \Lambda_{p}^{k}}\right)\right)= \\
& =\prod_{k=0}^{\infty} \prod_{\{p\}}\left(1-\frac{z^{n_{p}} \mathrm{e}^{\beta \sigma_{p}}}{\left|\Lambda_{p}\right| \Lambda_{p}^{k}}\right)
\end{aligned}
$$

Thus the spectral determinant may be expressed by an infinite product over the so called generalized zeta functions:

$$
\begin{aligned}
& \zeta_{(k) \beta}^{-1}(z)=\prod_{\{p\}}\left(1-\frac{z^{n_{p}} \mathrm{e}^{\beta \sigma_{p}}}{\left|\Lambda_{p}\right| \Lambda_{p}^{k}}\right) \\
& F_{\beta}(z)=\prod_{k=0}^{\infty} \zeta_{(k) \beta}^{-1}
\end{aligned}
$$


and the leading zero can be calculated from the zero order zeta, the dynamical zeta function $\zeta_{(0) \beta}^{-1}$.

We stress again that the quantities that enter the definition of zeta functions are the prime period $n_{p}$ of the orbit $p$, its instability $\Lambda_{p}=\prod_{i=0}^{n_{p}-1} \hat{f}^{\prime}\left(\hat{f}^{i}\left(x_{p}\right)\right.$ and the integer factor $\sigma_{p}$, that accounts for the orbit's behaviour once we unfold it on the real line. As a matter of fact, given any point $x_{p}$ belonging to $p\left(i . e . \hat{f}^{n_{p}}\left(x_{p}\right)=x_{p}\right.$ ) we may either have that it is also a periodic point of the lift $f\left(f^{n_{p}}\left(x_{p}\right)=x_{p}\right)$, or it might be a running mode, i.e. $f^{n_{p}}\left(x_{p}\right)=x_{p}+\sigma_{p}$, with $\sigma_{p} \in \mathbb{Z}^{*}($ see (14) $)$.

It is well known that zeta functions calculation may be recast in terms of perturbative expansions (the order being determined by the longest prime period available), that yield exponentially good estimates [4]. In order to show that let's use a simplified example: suppose there is a complete grammar in the simple alphabet $\{0,1\}$, calling for shortness $t_{p}=\frac{z^{n_{p}} \mathrm{e}^{\beta \sigma_{p}}}{\left|\Lambda_{p}\right| \Lambda_{p}^{k}}$ we can expand the infinite product in series:

$$
\begin{aligned}
\zeta_{(k) \beta}^{-1}(z)= & \prod_{\{p\}}\left(1-t_{p}\right)= \\
= & \left(1-t_{0}\right) \cdot\left(1-t_{1}\right) \cdot\left(1-t_{01}\right) \cdot\left(1-t_{001}\right) \cdot\left(1-t_{011}\right) \cdots \\
= & 1-t_{0}-t_{1}+ \\
& -\left(t_{01}-t_{0} t_{1}\right)-\left(t_{001}-t_{0} t_{01}\right)-\left(t_{110}-t_{1} t_{01}\right)-\cdots
\end{aligned}
$$

We can notice that there are fundamental terms $t_{0}$ and $t_{1}$ while others curvature term are made of a prime cycle shadowed by combinations of shorter cycles. Completely hyperbolic systems with a finite grammar yield curvature corrections that are exponentially small with the maximum period, so that (31) (power law expansion of the zeta function) is indeed a perturbative series.

We now sketch the technique we will actually use to investigate arbitrary order transport moments: first we reorder the zeros $\bar{z}_{i}$ of the spectral determinants so that the dominant ones come first and write in general:

$$
F_{\beta}\left(\mathrm{e}^{-s}\right)=\prod_{i}\left(1-\bar{z}_{i} \mathrm{e}^{-s}\right)
$$

so that

$$
\frac{d}{d s} \ln F_{\beta}\left(\mathrm{e}^{-s}\right)=\sum_{i} \frac{\bar{z}_{i} \mathrm{e}^{-s}}{\left(1-\bar{z}_{i} \mathrm{e}^{-s}\right)}
$$

and if we now take the inverse Laplace transform, we get

$$
\frac{1}{2 \pi \mathrm{i}} \int_{a-\mathrm{i} \infty}^{a+\mathrm{i} \infty} d s \mathrm{e}^{s n} \frac{d}{d s} \ln \left[F_{\beta}\left(\mathrm{e}^{-s}\right)\right]=\sum_{i} \bar{z}_{i}^{n}
$$

As the asymptotic behaviour is dominated by the leading eigenvalue we may extract it by using the dynamical zeta function instead of the spectral determinant, thus

$$
\bar{z}_{0}^{n}(\beta) \sim \frac{1}{2 \pi \mathrm{i}} \int_{a-\mathrm{i} \infty}^{a+\mathrm{i} \infty} d s \mathrm{e}^{s n} \frac{d}{d s} \ln \left[\zeta_{(0) \beta}^{-1}\left(\mathrm{e}^{-s}\right)\right]
$$


Remember that by probability conservation we have $\bar{z}_{0}(0)=1$ and all transport properties are captured by small $\beta$ expansion (11):

$$
\begin{aligned}
\sigma_{k}(n) & =\left\langle\left(x_{n}-x_{0}\right)^{k}\right\rangle_{0}=\left.\frac{\partial^{k}}{\partial \beta^{k}} G_{n}(\beta)\right|_{\beta=0} \\
& \left.\sim \frac{\partial^{k}}{\partial \beta^{k}} \frac{1}{2 \pi \mathrm{i}} \int_{a-\mathrm{i} \infty}^{a+\mathrm{i} \infty} d s \mathrm{e}^{s n} \frac{d}{d s} \ln \left[\zeta_{(0) \beta}^{-1}\left(\mathrm{e}^{-s}\right)\right]\right|_{\beta=0}
\end{aligned}
$$

The evaluation of the integral on the right hand side of (36) requires dealing with high order derivatives of a composite function: this is generally accomplished by making use of Faà di Bruno formula:

$$
\begin{aligned}
& \frac{d^{n}}{d t^{n}} H(L(t))=\sum_{k=1}^{n} \sum_{k_{1}, \ldots, k_{n}} \frac{n !}{k_{1} ! \cdots k_{n} !} \frac{d^{k} H}{d L^{k}}(L(t)) \cdot B_{\boldsymbol{k}}(L(t)) \\
& B_{\boldsymbol{k}}(L(t))=\left(\frac{1}{1 !} \frac{d L}{d t}\right)^{k_{1}} \cdots\left(\frac{1}{n !} \frac{d^{n} L}{d t^{n}}\right)^{k_{n}} \\
& \boldsymbol{k}=\left\{k_{1}, \ldots k_{n}\right\} \text { with } \sum k_{i}=k, \quad \sum i \cdot k_{i}=n
\end{aligned}
$$

Remember that the characterization of anomalous transport is captured by the function $\nu(q)$ (the fundamental quantity we want to evaluate) that expresses the asymptotic growth of moments of arbitrary order:

$$
\left\langle\left|x_{t}-x_{0}\right|^{q}\right\rangle \sim t^{\nu(q)}
$$

We already mentioned how ordinary diffusion yields $\nu(q)=q / 2$, while anomalous transport typically shows a non trivial behaviour, which cannot be encoded by a single exponent, but rather typically exhibits a phase transition [15, 12, 16. In next sections we will apply the formalism to both normal and anomalous examples. These computations accomplish a twofold goal: besides checking the theory, they will also illustrate how subtle features of the underlying dynamical system are automatically included in the formalism.

\section{Normal transport}

We now show how the technique is able to reproduce the well known exponents $q / 2$ for the transport moments of a completly chaotic dynamic. This oversimplified example is a warmup exercise before facing more involved (and interesting) cases.

Consider the following map on the real line

$$
f(x)= \begin{cases}a x & x \in[0,1 / 2] \\ a(x-1)+1 & x \in(1 / 2,1]\end{cases}
$$

For a generic value of the parameter a symbolic dynamics of (41) is quite complicated (and this leads to interesting consequences, like fractal dependence of the diffusion constant on the slope $a$ [17]): on the contrary by choosing an integer slope the torus map consists of complete branches: for example for $a=2 m, m \in \mathbb{N}$ the corresponding 
torus map consists of $2 m$ complete branches and the grammar is thus unrestricted in a $2 m$ alphabet. Moreover the linearity of the map leads to complete cancellations of the curvature terms: whenever the weights associated to each cycle depend multiplicatively on the symbolic code entries, the contribution of a cycle and corresponding pseudocycles cancel out exactly and zeta functions pick up contribution only from fixed points.

In this case there are $2 m$ fixed points: the instabilities are trivially $\Lambda_{i}=a$ for every fixed point while the jumping numbers associated to each branch are clearly: $0,1, \cdots,(a-1),-(a-1), \cdots,-1,0$.

The zeta function for the torus map is then:

$$
\zeta_{0\{\beta\}}^{-1}(z)=1-\frac{2 z}{a}\left(1+\sum_{k=1}^{a / 2-1} \cosh (\beta k)\right)
$$

Long time behaviour of the q-th moment is captured by (36). Following [4] we concentrate first on $\beta$ derivatives

$$
\left.\frac{\partial^{n}}{\partial \beta^{n}} \ln \zeta_{0\{\beta\}}^{-1}(z)\right|_{\beta=0}
$$

Faà di Bruno's expansion (37) generates:

$$
\left.\sum_{k=1}^{n} \sum_{k_{1}, \cdots k_{n}} \frac{1}{\zeta_{0\{\beta\}}^{-1}(z)^{k}} \cdot B_{\boldsymbol{k}}(z)\right|_{\beta=0}
$$

and a generic term contributing to the product in $B_{\boldsymbol{k}}(z)$ is now

$$
\frac{\partial^{k_{j}}}{\partial \beta^{k_{j}}} \zeta_{0\{\beta\}}^{-1}(z)_{\mid \beta=0}= \begin{cases}\frac{2 z}{a} \sum_{l=1}^{a / 2-1} l^{k_{j}} & k_{j} \text { even } \\ 0 & k_{j} \text { odd }\end{cases}
$$

Each term in (44) has a different asymptotic behaviour and contribute differently to the transport moment once we perform the derivative with respect to $s$ and take the inverse Laplace transform in (36). The long time behaviour of the q-th transport moment is captured by choosing the leading singularity of the $\beta$ derivatives near $z=1(s=0)$.

The numerator in (44) doesn't diverge for $z \rightarrow 1$ and the divergence comes only from the denominator:

$$
\frac{1}{\zeta_{0\{\beta\}}^{-1}(z)^{k}} \sim(1-z)^{-k}
$$

The leading singularity comes from the term with the largest possible choice for $k$ and hence all $k_{j}=2$ (remember that for $k_{j}$ odd the numerator vanish). This leads to the estimate

$$
\frac{\partial^{n}}{\partial \beta^{n}} \ln \zeta_{0\{\beta\}}^{-1}(z)_{\mid \beta=0} \sim(1-z)^{-n / 2}
$$

and, performing the inverse Laplace transform, this yields $\nu(q)=q / 2$, typical of normally diffusing systems (where one is then interested in obtaining estimates of the prefactor, namely the diffusion constant). We turn now on more interesting case of intermittent diffusion where the presence of marginal points deeply modified the structure of transport exponents. 


\section{Intermittent systems}

In the last few years it has been realized [18, 19, 20, 21, 22] that weakly chaotic systems (in particular one dimensional intermittent maps, or infinite horizon Lorentz gas models lead to more complicated analytic structure of zeta functions, which typically exhibit branch points. In view of the inverse Laplace formula (36) the modified analytic structure of the zeta function may induce anomalous behaviour (nonlinear diffusion [23, 4]).

The qualitative difference between an hyperbolic system and an intermittent one can be well appreciated in fig. (11) where a long segment of an orbit for both cases is plotted against time.
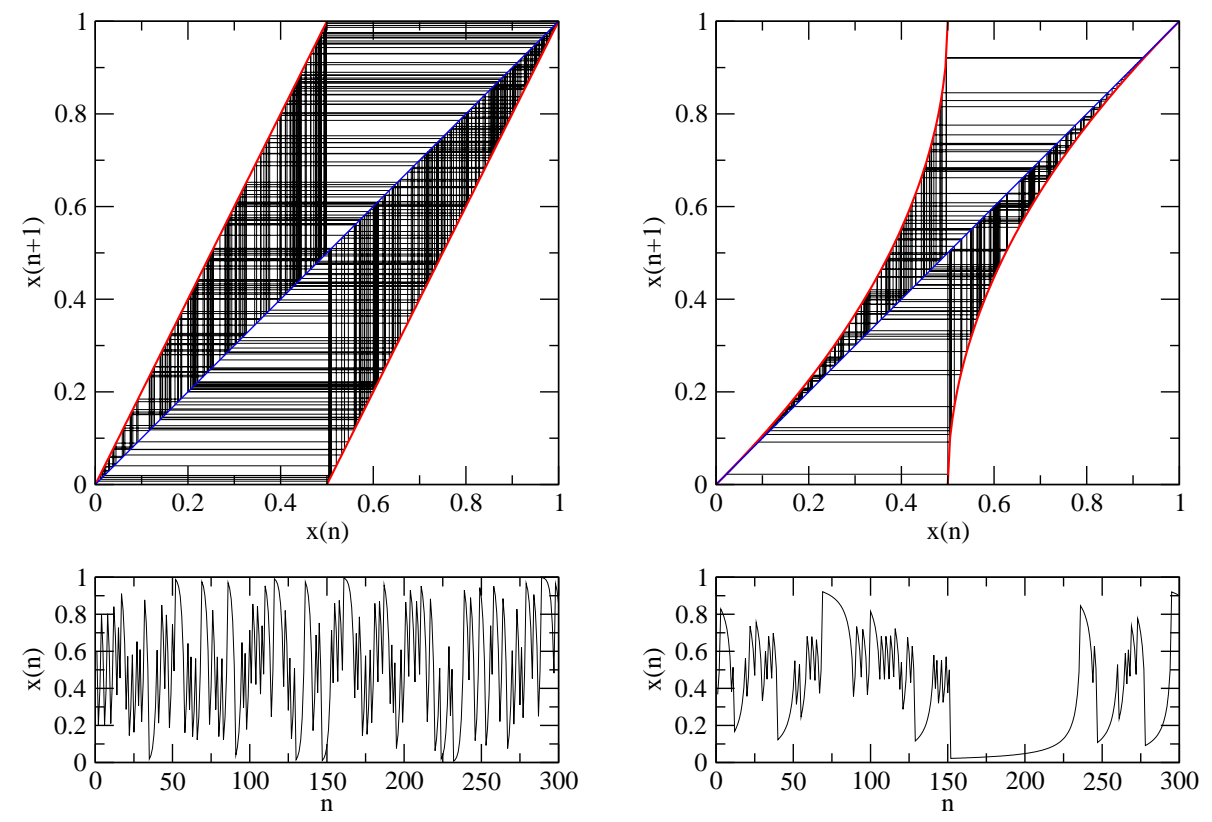

Figure 1. A segment of trajectory (300 iterations) with the same initial condition is showed for two torus maps: a completely hyperbolic map (left) and one with intermittent fixed points (right).

In the intermittent case chaotic wandering is interrupted by intervals of regular motion, generated by points that start their motion near the marginal point and slowly move away from it: we will see that there is a power low separation for nearby trajectories instead of the exponential growth we normally expect. In the next subsection we will use the thermodynamic formalism in order to detect the presence of power law behaviour in instabilities spectrum while in the following subsections we explain how it is possible to implement cycle expansion also in this anomalous case.

\subsection{Thermodynamic formalism}

In order to obtain information about the scaling behaviour of periodic orbits instabilities we turn our attention to a thermodynamic analysis of this set. Consider all periodic 
points of $n$ order $x_{i(n)}, i=1 \ldots N_{n}$. Consider now the partition of the unit interval with the intervals:

$$
I_{i(n)}=\left(x_{i(n)}, x_{i+1(n)}\right) \quad i=1 \ldots N_{n}
$$

whose length we denote by $l_{i(n)}=\left|I_{i(n)}\right|$, and construct with them the following partition function:

$$
Z_{n}(\tau)=\sum_{i=1}^{N_{n}-1}\left(l_{i(n)}\right)^{-\tau}
$$

where the parameter $\tau$ is the formal analogue of an inverse temperature. By varying $\tau$ one probes for all the relative different scaling of the intervals in the partition function sum [24]. For example for $\tau \rightarrow+\infty$ the partition function will be dominated by the thinnest intervals while for $\tau \rightarrow-\infty$ the fattest ones prevail.

Scaling properties may be analyzed [25] by writing

$$
l_{i(n)}=\left(N_{n}\right)^{-\mu_{i(n)}}
$$

where we have introduced the local scaling indices $\mu_{i(n)}$ : it is important to notice that they will be finite if the interval shrink exponentially with $n$ (as typically $N_{n} \sim a^{n}$ ) while will go to zero as $(\ln n) / n$ for a power law behaviour.

The (asymptotic) free energy is then defined as

$$
\begin{aligned}
g(\tau) & =\lim _{n \rightarrow \infty} \frac{1}{\ln \left(N_{n}\right)} \ln Z_{n}(\tau) \\
& =\lim _{n \rightarrow \infty} \frac{1}{\ln \left(N_{n}\right)} \ln \sum_{i} N_{n}^{\tau \mu_{i(n)}}
\end{aligned}
$$

from which we can extract the average scaling index:

$$
\mu(\tau)=g^{\prime}(\tau)=\lim _{n \rightarrow \infty} \frac{\sum_{i} \mu_{i(n)} N_{n}^{\tau \mu_{i(n)}}}{\sum_{i} N_{n}^{\tau \mu_{i(n)}}}
$$

Figure (2) shows four approximated scaling index functions $\mu_{n}(\tau)$ calculated for an intermittent map: this map has two complete branches and thus the number of periodic points of order $n$ grows as $2^{n}$ while the presence of marginal points generates a family of intervals that shrink polynomially with $n$ (the exact form of this map will be presented as second example in following subsection (see (76)). We can note that by increasing the hierarchical order the scaling index converge to finite values for $\tau>0$ while it slowly goes to zero for $\tau<0$, as we expect due to intervals that shrink with power law behaviour.

The thermodynamic analysis may be pushed further by rewriting the partition function as:

$$
Z_{n}(\tau)=\int_{\mu_{\min }}^{\mu_{\max }} \mathrm{d} \mu N_{n}^{\left(s_{n}(\mu)+\mu \tau\right)}
$$

where $N_{n}^{s_{n}(\mu)}$ is the density of intervals with scaling index $\mu$. As the number of periodic orbits increases the integral can be approximated by the saddle point method:

$$
N_{n}^{g_{n}(\tau)}=C(n)\left[-\frac{2 \pi \ln N_{n}}{S_{n}^{\prime \prime}(\tau)}\right]^{\frac{1}{2}} N_{n}^{\left(S_{n}(\bar{\mu})+\tau \bar{\mu}\right)}
$$




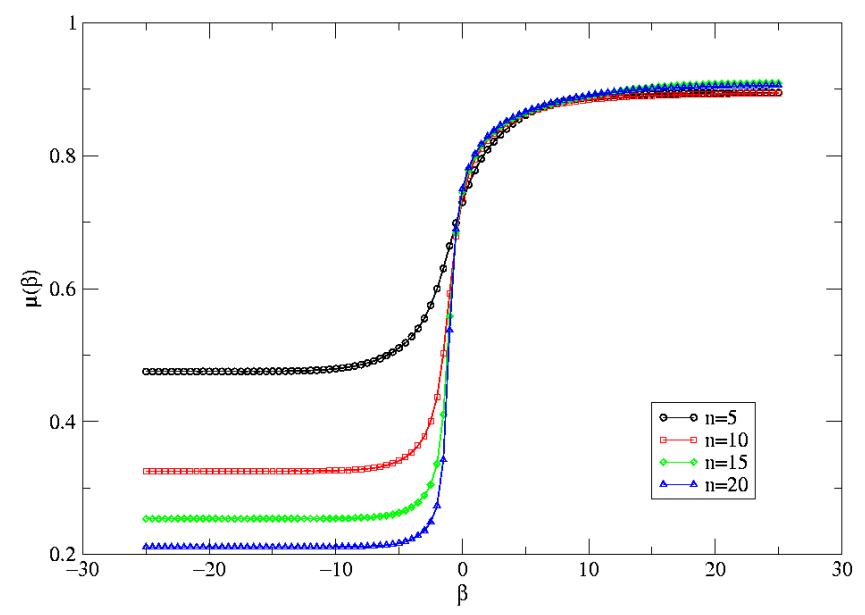

Figure 2. Approximated scaling index functions $\mu_{n}(\tau)$ versus $\tau$ for the map in (76) with $\gamma=2.5$ and with $\mathrm{n}=5,10,15,20$.

where $\bar{\mu}$ is the solution of the extremum condition

$$
\left.\frac{d S_{n}(\tau)}{\mathrm{d} \mu}\right|_{\bar{\mu}}=-\tau
$$

and $g_{n}(\tau)$ is the n-th order approximation to the free energy.

The scaling spectrum $s_{n}(\mu)$ is a highly irregular function of $\mu$ for finite $n$ : in practise one evaluates $g_{n}(\tau)$ and replaces $s(\mu)=\lim _{n \rightarrow \infty} s_{n}(\mu)$ by its convex envelope $S(\mu)$, evaluated in the thermodynamic limit saddle point evaluation of (54).

For the very same example used in calculating the scaling index of figure (2) we now compute the convex envelope entropy $S_{n}(\mu)$. While for a complete hyperbolic dynamic we expect a convergence to a curve with strictly positive support, in our case marginality yields a tail in the small scaling region of $S(\mu)$, which slowly advances towards zero as the hierarchical order increases, see figure (3): the right branch of entropy is stable and it is representative of exponential scalings while the leftmost branch slowly moves towards zero, being ruled by intervals shrinking with power laws.

\subsection{Transport}

We now apply the technique to two classes of one dimensional maps, where deviations from fully chaotic behaviour are provided by marginal fixed points of intermittent type [26]. The prototype example of this class of systems is provided by the mapping on the unit interval

$$
x_{n+1} \sim x_{n}+\left.x_{n}^{\gamma}\right|_{\bmod 1}
$$

where $\gamma>1$ is called the intermittency exponent. 


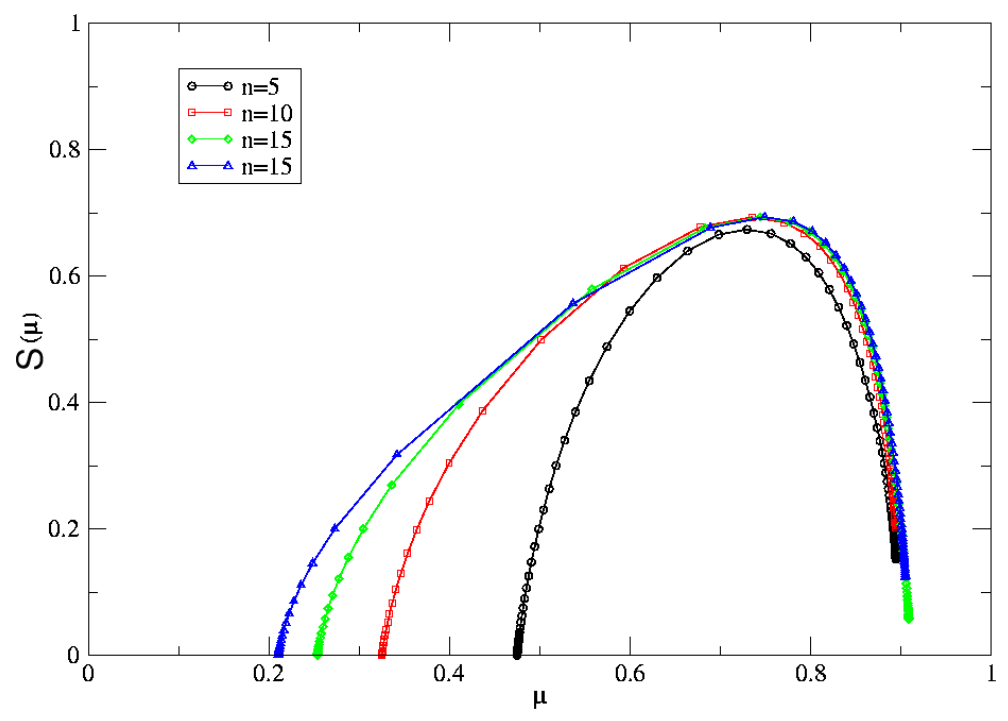

Figure 3. Approximated entropies $S_{n}(\mu)$ versus $\mu$ for the map (76) with $\gamma=2.5$ and with $\mathrm{n}=5,10,15,20$.

The presence of the marginal fixed point causes problems in using zeta function techniques in a straightforward manner: this fixed point has $|\Lambda|=1$, which leads to divergences (see (21)). The way out of this problem is to prune the $\overline{0}$ fixed point away: from a symbolic dynamics point of view this leads to an infinite alphabet, since all $0^{n} 1$ fixed points lack curvature counterterms: the fundamental cycle part of the zeta function thus becomes (cfr. (31))

$$
\zeta_{\text {fund }}^{-1}(z)=1-t_{1}-t_{01}-t_{0^{2} 1}-\cdots-t_{0^{n} 1}-\cdots
$$

so even if curvature corrections are neglected we do not have a finite order polynomial: moreover the inclusion of curvature correction even in simple cases is by no means trivial 19. As we will see by considering specific examples, the analytic structure of (158) will be strongly dependent on the intermittency exponent, through the behaviour of stability of cycles $0^{n} 1$ coming closer and closer to the marginal fixed point.

\subsection{Intermittent diffusion I}

We can turn now on a specific example. The symmetry requirements are the same as the completely chaotic example (see relations (12) and (13)).

The map on the fundamental cell (see figure (44) consists of two intermittent branches and one central hyperbolic region. If we assign jumping numbers $\sigma_{L}=-1$ to the left branch, $\sigma_{C}=0$ to the central one and $\sigma_{R}=+1$ to the right branch we get the lift map of figure (4). Whenever a particle remain trapped near a marginal point the corresponding unfolded trajectory on the real line consists of successive jumps on the neighbouring cell.

The presence of complete branches leads to an unrestricted grammar in the symbolic code $\{L, C, R\}$, where the corresponding partition obviously consist of the inverse image 

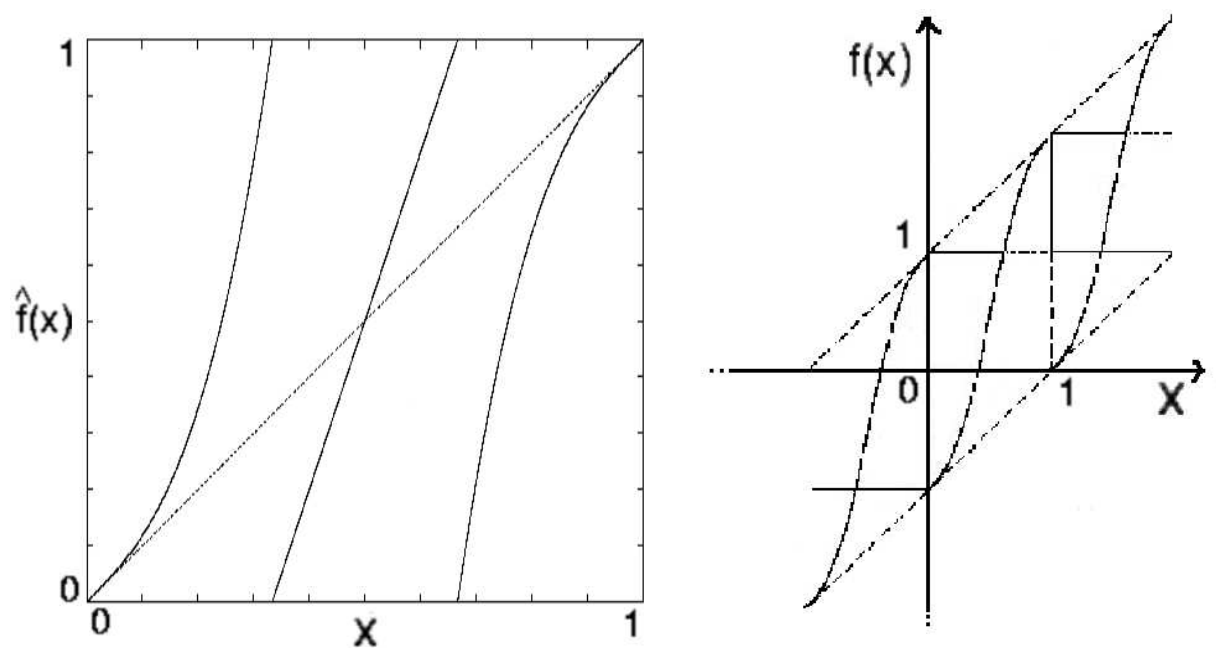

Figure 4. A torus map with intermittent fixed points, and the corresponding lift.

of the unit interval under the corresponding three branches functions $f_{L}, f_{C}, f_{R}$. However we have to prune marginal fixed points $\bar{L}$ and $\bar{R}$ away, as explained before: the grammar is thus unrestricted on a countable new alphabet:

$$
\left\{C, L^{i} C, L^{j} R, R^{k} C, R^{l} L\right\} \quad i, j, k, l \in \mathbb{N}_{+}
$$

The boundary points of such a partition can be constructed by sequences of iterates of the inverse map ( $\mathrm{L}$ or $\mathrm{R}$ branches): $f_{L}^{-1}(1), f_{L}^{-2}(1), \ldots, f_{R}^{-1}(0), f_{R}^{-2}(0), \ldots$. The corresponding zeta function will thus consist of a (non polynomial) fundamental part (58), together with curvature corrections: by a straightforward generalization of Gaspard-Wang piecewise linear approximation [27] of Pomeau-Manneville map, we can obtain an approximate model where all curvature terms vanish and the zeta function can be explicitly written without losing important dynamical features [28]. The power law scaling of the stability of cycles coming closer and closer to the marginal points can be extimated as

$$
\Lambda_{i} \sim i^{-(\alpha+1)}
$$

where $i$ indicates the number of iteration the orbits needs to escape from the laminar phase, and

$$
\alpha=1 /(\gamma-1)
$$

$\gamma$ being the intermittency exponent of the L-R branches (see (57)).

The zeta function is:

$$
\zeta_{(0) \beta}^{-1}(z)=1-a z-b z \sum_{k=1}^{\infty} \frac{z^{k}}{k^{\alpha+1}} \cosh (\beta k)
$$

where $a$ and $b$ are fixed by specifying the central region slope and the normalization condition $\zeta_{(0) 0}^{-1}(1)=0$. 
When equipped with information about the analytic structure of the zeta function, from (37) we may single out the leading singularity in the logarithmic derivative of the zeta function, and then estimate the asymptotic behaviour of (36). In our previous example the only divergence for $z \rightarrow 1$ in derivatives expansions (37) comes from the denominator while here the appearance of the Bose function (see (62))

$$
g_{\mu}(z)=\sum_{l=1}^{\infty} \frac{z^{l}}{l^{\mu}}
$$

indeed may alter the analytic features. We recall how the behaviour as $z \rightarrow 1^{-}$depends upon $\mu$ (related to the intermittency exponent)

$$
g_{\mu}(z) \sim \begin{cases}(1-z)^{\mu-1} & \mu<1 \\ \ln (1-z) & \mu=1 \\ \zeta(\mu)+C_{\mu}(1-z)^{\mu-1}+D_{\mu}(1-z) & \mu \in(1,2) \\ \zeta(2)+C_{2}(1-z) \ln (1-z) & \mu=2 \\ \zeta(\mu)+C_{\mu}(1-z) & \mu>2\end{cases}
$$

We stress again that this functions appear as a consequence of particular sequences of orbits coming closer and closer to the marginal fixed points whose stability increases only polynomially with the period [27, 4, a clear signature of local deviation from typical hyperbolic behaviour (which is ruled by exponential instability growth).

Now look at a generic term in (44) and denote it by $\mathcal{D}_{k_{1} \ldots k_{n}}$. Taking into account that

$$
\left.\frac{\partial^{i}}{\partial \beta^{i}} \zeta_{(0) \beta}^{-1}(z)\right|_{\beta=0} \sim \begin{cases}0 & i \text { odd } \\ z g_{\alpha+1-i}(z) & i \text { even }\end{cases}
$$

we have that

$$
\mathcal{D}_{k_{1} \ldots k_{n}} \sim \frac{1}{\left(\zeta_{(0) 0}^{-1}(z)\right)^{k}} \prod_{j}\left(g_{\alpha+1-j}(z)\right)^{k_{j}}=\frac{\mathcal{D}_{k_{1} \ldots k_{n}}^{+}}{\mathcal{D}_{k_{1} \ldots k_{n}}^{-}}
$$

where the $\mathcal{D}^{+}$picks up the contributions from the product of Bose functions, and all $j$ must be even, due to (65). First we consider the case $\alpha \in(0,1)$, which corresponds to intermittency exponent $\gamma>2$ : we have $\mathcal{D}_{k_{1} \ldots k_{n}}^{-} \sim(1-z)^{k \alpha}$, that, together with (64), implies that the dominant singularity is of the form

$$
\mathcal{D}_{n} \sim \frac{1}{(1-z)^{\rho}}
$$

where $\rho$ is determined by

$$
\rho=\sup _{\left\{k_{1} \ldots k_{n}\right\}}\left(k \alpha+\sum_{j}(j-\alpha) k_{j}\right)=n
$$

Once plugged into (36) this leads to the estimate

$$
\nu(q)=q
$$

which means that the whole set of transport moments is ruled by ballistic behaviour (at least for even exponents, where the method applies). We now turn to the more subtle 
case $\alpha>1$ : since the dynamical zeta function has a simple zero we get $\mathcal{D}_{k_{1} \ldots k_{n}}^{-} \sim(1-z)^{k}$, while the terms appearing in $\mathcal{D}^{+}$modify the singular behaviour near $z=1$ only for sufficiently high $j$

$$
g_{\alpha+1-j}(z) \sim \begin{cases}(1-z)^{\alpha-j} & j>\alpha \\ \zeta(\alpha+1-j) & j<\alpha\end{cases}
$$

If all $\{j\}$ are less than $\alpha$ then the singularity is determined by $\mathcal{D}^{-}$: keeping in mind that the highest $k$ value is achieved by choosing $j=2$ and $k_{2}=n / 2$, we get, by proceeding as before

$$
\nu(q)=\frac{q}{2} \quad q<\alpha
$$

When $q$ exceeds $\alpha$ we have to take into account possible additional singularities in $\mathcal{D}^{+}$, and thus we get

$$
\mathcal{D}_{n} \sim \frac{1}{(1-z)^{\rho}}
$$

where $\rho$ is determined by

$$
\rho=\sup _{\left\{k_{1} \ldots k_{n}\right\}}\left(k+\sum_{j>\alpha}(j-\alpha) k_{j}\right)= \begin{cases}n / 2 & n<2(\alpha-1) \\ n+1-\alpha & n>2(\alpha-1)\end{cases}
$$

which, once we take (71) into account, yields

$$
\nu(q)= \begin{cases}q / 2 & q<2(\alpha-1) \\ q+1-\alpha & q>2(\alpha-1)\end{cases}
$$

The set of exponents thus has a nontrivial structure, characterized by a sort of phase transition for $q=2(\alpha-1)$, a rather universal feature of many systems exhibiting anomalous transport [12, 16]. We notice that the parameter ruling the presence of a phase transition (and the explicit form of the spectrum) is $\alpha$, that is the exponent describing the polynomial instability growth of the family of periodic orbits coming closer and closer to the marginal fixed point, and thus describing the sticking to the regular part of the phase space: in the present example $\alpha=(\gamma-1)^{-1}$, and thus the sticking exponent is easily connected to the intermittency index. We remark that our reference to "phase transitions" is somehow arbitrary, since in effect our technique applies in the present form only to even moments: however results as (174) are consistent with all our numerical checks for the whole real $q$ axis.

\subsection{Intermittent diffusion II}

We now discuss a further example, which will provide additional evidence of the virtues of our technique. While the setting is similar to the former case we slightly modify it to conform to original references. The map we consider belongs to a class of 2-dimensional systems were the diffusive process takes place in a direction while all the dynamics is 
limited to the torus on the other dimension:

$$
\left\{\begin{array}{l}
x_{t+1}=\hat{f}\left(x_{t}\right) \\
y_{t+1}=y_{t}+x_{t}
\end{array}\right.
$$

where $\hat{f}(x)$ is a torus map, again dependent on an intermittency parameter $\gamma$, implicitly defined on $\mathcal{T}=[-1,1)$ in the following way [15]:

$$
x= \begin{cases}\frac{1}{2 \gamma}(1+\hat{f}(x))^{\gamma} & 0<x<1 /(2 \gamma) \\ \hat{f}(x)+\frac{1}{2 \gamma}(1-\hat{f}(x))^{\gamma} & 1 /(2 \gamma)<x<1\end{cases}
$$

for negative values of $x$ the map is defined as $\hat{f}(-x)=-\hat{f}(x)$ (see (13)): the map is plotted in fig. (5). Diffusion can be treated by the same techniques of the former subsection if we are able to control the values the function (equivalent to the jumping numbers of previous example)

$$
\sigma_{p}=\sum_{t=1}^{T_{p}} x_{t}
$$

takes on the periodic orbits.

The most striking difference with respect to the former example is that, for any values of $\gamma$, the map (76) has a constant invariant measure [15].

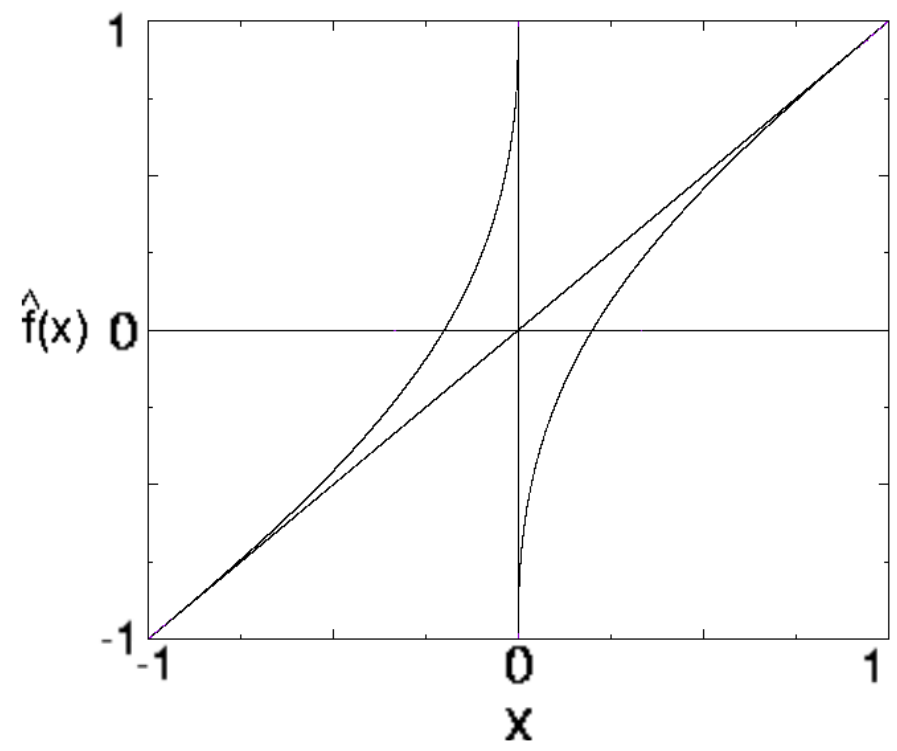

Figure 5. The intermittent map with constant invariant measure.

This comes from the summation property

$$
\sum_{y=\hat{f}^{-1}(x)} \frac{1}{\hat{f}^{\prime}(y)}=1
$$


that easily follows once we observe that each point has exactly two counter images $y_{1}(x)$ and $y_{2}(x)$ : they come from two different pieces in (76) and they have opposite sign: by deriving the two pieces of (76) then we get the summation rule. This is quite different from the former case where the torus map has non trivial ergodic properties: an absolutely continuous invariant measure only exists for $\alpha>1$ (see for instance 29] and references therein) while the ergodic behaviour is much more complex when $\alpha<1$. In any case sticking induces peaking of the measure around marginal fixed points (see [27]). In this sense the behaviour of (76) is similar to what happens in an area preserving map example [30, where a parabolic fixed point coexist with a Lebesgue invariant measure.

The difference with the previous example can be euristically understood if we note that now the slope of the map in the chaotic region is not bounded from above: the closer we need to be injected near the marginal points the lesser the probability that this happens. This feature deeply modifies the instabilities of periodic orbits coming closer and closer to the marginal fixed points and we expect that a linearization as before will not be able to reproduce them.

A linearization is still possible but we must put attention on matching the condition (178). To do that let's start by identifying for each branch a laminar and an injection regions (for example we call $\overline{\mathrm{B}}=[0,1 /(2 \gamma)]$ and $\mathrm{B}=[1 /(2 \gamma), 1]$ respectively the injection and laminar regions of the right branch (see figure (6) ).

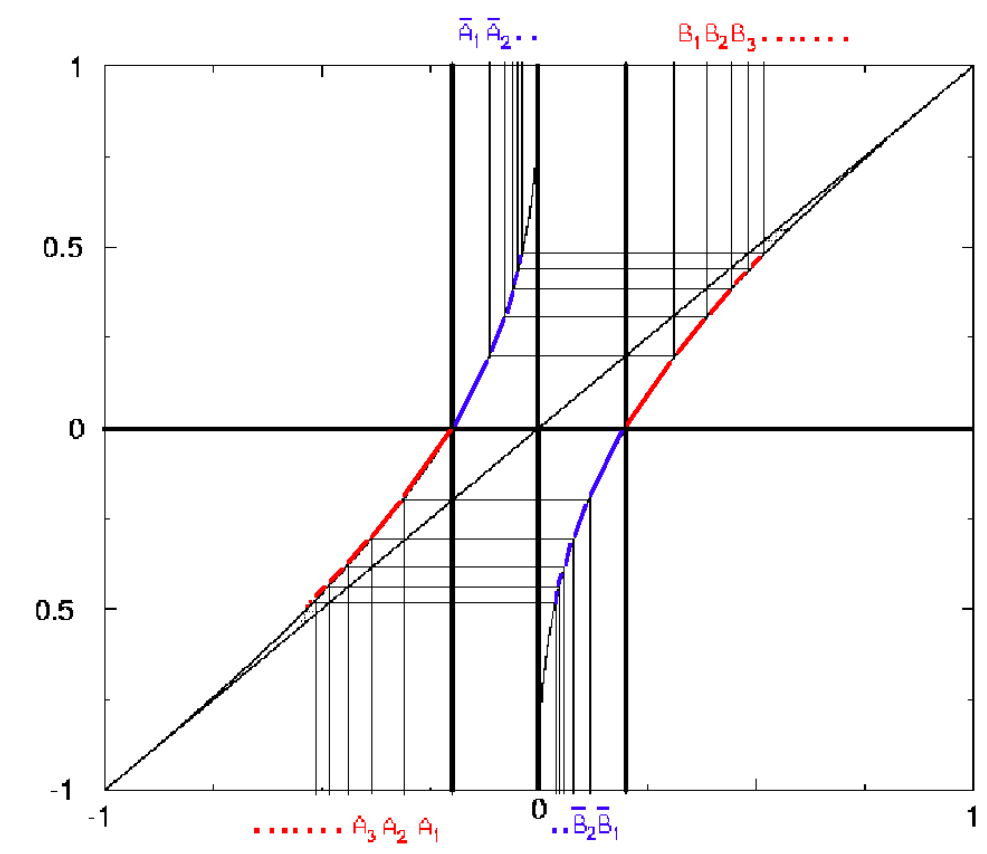

Figure 6. Linearization of the map (76). Near marginal points, laminar regions, a Gaspard-Wang type partition is used while a finer one is used in the injection zone .

The Gaspard-Wang linearization has to be modified in the injection zones $\overline{\mathrm{A}}$ and $\overline{\mathrm{B}}$ : a linear approximation for all such intervals will give an uniform probability of being reinjected in the laminar zone and this does not respect the peculiar ergodic 
property of this map. The way out to this problem is to perform a finer partition of that regions taking the inverse image (inside the injection zones) of each interval partitioning the corresponding laminar zone. For example we will call $\overline{\mathrm{B}}_{1}$ the injection interval corresponding to inverse image of the laminar one $A_{1}, \bar{B}_{2}$ corresponding to $A_{2}$ and so on (see figure (6i)). The linearization is now straightforward for each of this intervals. Moreover the instabilities of all the periodic orbit are well reproduced once we note that the slope of the linearized map restricted to such injection intervals can be safely approximated by (see summation property (78)):

$$
\Lambda_{\overline{\mathrm{B}}_{i}}^{-1}=1-\Lambda_{\mathrm{A}_{i}}^{-1}
$$

We will now show how such a linearization is able to reproduce the behaviour of periodic orbit instabilities. We have seen that the periodic orbits that are relevants for the construction of the zeta function are the ones that accumulate near the marginal fixed point and for which the instability has a power law behaviour. In this new map the instability of such orbits take a linear correction factor (with respect to the length) from the injection zone: the intervals in the laminar regions, constructed in the same spirit of Gaspard-Wang linearization [27], shrink polinomially with the index $k$ :

$$
l_{k} \sim \frac{C}{k^{\alpha+1}}
$$

from wich is simple to derive the periodic orbit instabilities. We rewrite the instabilities of the relevant orbits for the previous example (I) in comparison with the ones of this map (II):

$$
\begin{aligned}
\Lambda_{L^{k} R}^{I} & =\left[\frac{l_{0}}{l_{1}} \frac{l_{1}}{l_{2}} \ldots \frac{l_{k-1}}{l_{k}}\right]= \\
& \sim k^{\alpha+1} \\
\Lambda_{A^{k} B}^{I I} & =\left[\frac{l_{0}}{l_{1}} \frac{l_{1}}{l_{2}} \ldots \frac{l_{k-1}}{l_{k}}\right]\left[1-\frac{l_{k}}{l_{k-1}}\right]^{-1}\left[1-\frac{l_{1}}{l_{0}}\right]^{-1} \\
& \sim \Lambda_{L^{k}}^{I} \frac{l_{k-1}}{l_{k-1}-l_{k}} \sim k \Lambda_{L^{k} R}^{I} \\
& \sim k^{\alpha+2}
\end{aligned}
$$

where we note that for this second example the modified linearization produce two new factors (see (83)) that correspond to the slope of the two injection intervals visited by the orbit; one of them depends explicitly on the lenght of the orbit and gives a relevant correction to the instability of the cycle (84).

This trend is what we expect from numerical simulations (see figure (77)). Note that this new linearization is able to capture the behaviour of the instabilities of all orbits, not only the one accumulating to the marginal point. As a further example we may consider periodic orbits of the type $A^{k} B^{k}$ were the power law exponent is twice the one before, in agree with performed numerical simulations. Thus (78) deeply modifies the relationship between the intermittency exponent and the instabilities of periodic orbits shadowing the marginal fixed points. 


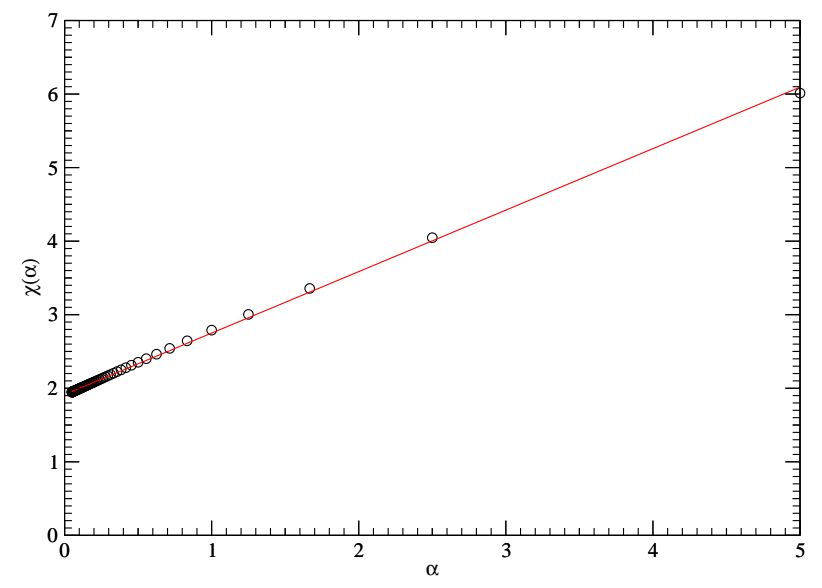

Figure 7. The exponent $\chi(\alpha)$ of power law decay of instabilities $\Lambda_{A^{k} B}^{I I} \sim k^{\chi(\alpha)}$ as a function of the parameter $\alpha$ (open circles) and the best fit $\chi(\alpha)=(0.88 \alpha+1.9)$ (full line).

Once we notice that, for the cycles dominating the zeta function expression, the jumping function (77) can be approximate by the lenght of the periodic orbit (with the appropriate sign), the piecewise linear approximation yields the dynamical zeta function

$$
\zeta_{(0) \beta}^{-1}(z)=1-\zeta(\alpha+2) z \sum_{k=1}^{\infty} \frac{z^{k}}{k^{\alpha+2}} \cosh (\beta k)
$$

where again $\alpha=1 /(\gamma-1)$.

By repeating the steps of the calculation performed in the former case we get that we always have a phase transition with

$$
\nu(q)= \begin{cases}q / 2 & q<2 \alpha \\ q-\alpha & q>2 \alpha\end{cases}
$$

which may also be checked numerically (see figure (8) ).

\subsection{The Lorentz gas with infinite horizon}

The technique does not only apply to one dimensional systems, but is capable of dealing with more complicated systems, if we are able to characterize the sequence of orbits that probe more and more accurately the local behaviour near the ordered region. A remarkable example in this respect is the Lorentz gas with infinite horizon (see fig. (9)), where a particle collides elastically with a regular array of fixed circular scatterers [3], the corresponding torus map being a square billiard table with a circular scatterer in the center (the so called Sinai billiard [3, 10]). In this example the dynamical feature that lowers the chaoticity of the system is provided by the possibility of infinite free flights along corridors (for instance horizontal and vertical direction in fig. (99)). Periodic orbits for the torus maps (running modes for the full system) that mimic the free flight 


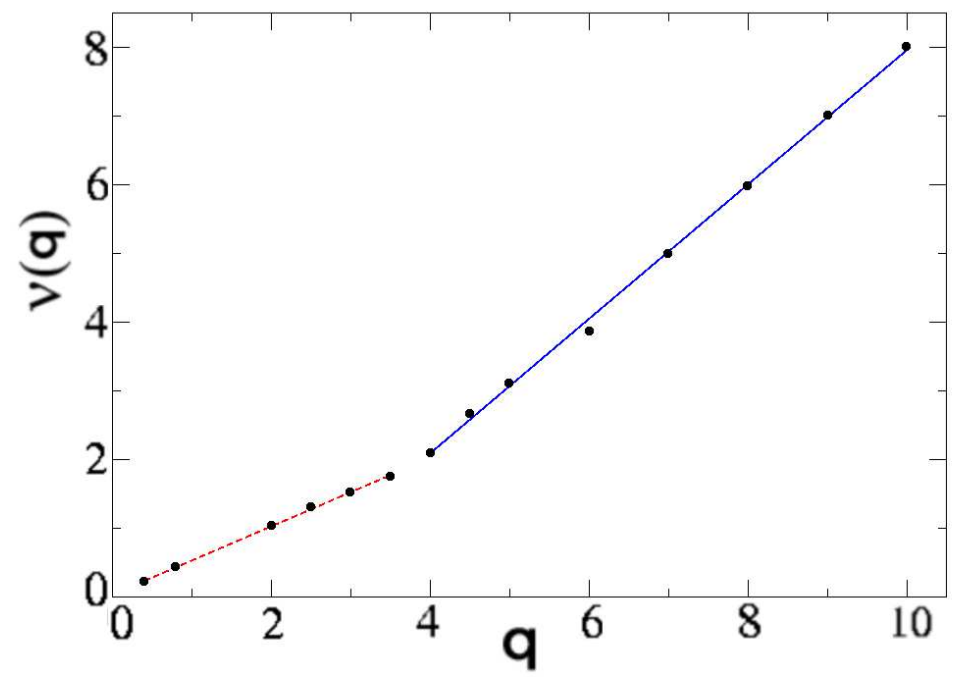

Figure 8. Spectrum of the transport moments for the map (76) with $\gamma=1.5$ : the best fit on numerical data is $\nu(q)=0.50 q+0.04$ for the dotted line, and $\nu(q)=0.98 q-1.82$ for the full line.

are for instance orbits that bounce from the $(m, 0)$ row to the $(n, 1)$ row travelling a distance of $p$ lattice spacings between each two successive collisions. In view of our

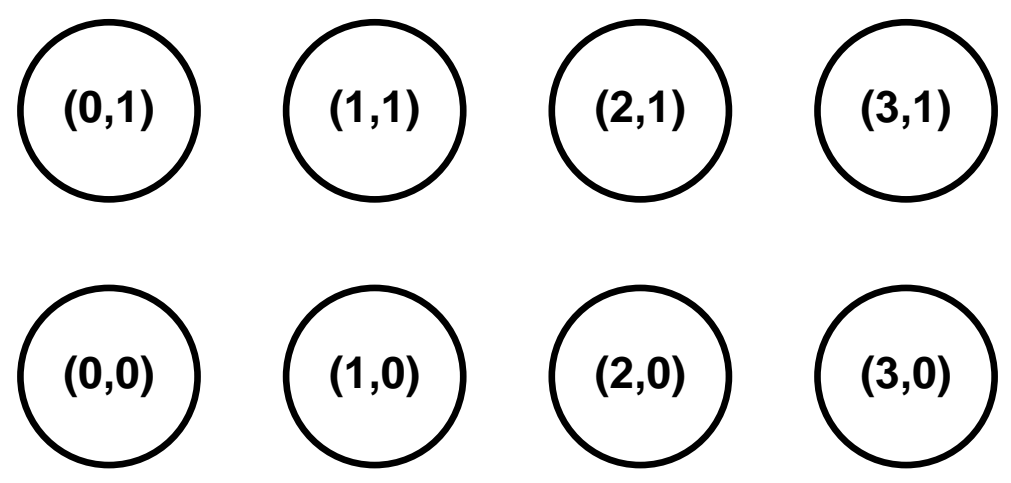

Figure 9. Unbounded horizon Lorentz gas, with discs labelling.

former considerations the estimate we need is how the stability of such orbits scales in the big $p$ limit. Such a calculation is equivalent to computing the fraction of orbits that starting from the $(0,0)$ disc suffer their first collision exactly in the $(p, 1)$ disk: such a probability decays like $1 / p^{3}$ (see [20]), and in this sense the Lorentz gas corresponds to the case $\alpha=2$, in (62). This leads to a direct estimate of the spectrum of exponents, according to (74):

$$
\nu(q)= \begin{cases}q / 2 & q<2 \\ q-1 & q>2\end{cases}
$$

It is interesting to show how the phase transition sits exactly at $q=2$, that is the order corresponding to diffusion. The behaviour described in (88), was obtained recently in 
[16] (actually for a wider class of extended billiards), by different methods. We also remark that our method is also capable of taking track of logarithmic corrections to the asymptotic estimates, and in the present example we get that the variance (second moment) asymptotically grows as $t \cdot \ln t$ (see [10]). The appearance of a logarithmic correction may be understood if we notice that, for $\alpha=2$, we have that

$$
\frac{\partial^{2}}{\partial \beta^{2}} \zeta_{(0) \beta}^{-1}\left(\mathrm{e}^{-s}\right) \sim \frac{\ln \left(1-\mathrm{e}^{-s}\right)}{\left(1-\mathrm{e}^{-s}\right)}
$$

(cfr. (62) ): then the leading singularity, dominating the integral (36) is of the form $\ln \left(1-\mathrm{e}^{-s}\right) /\left(1-\mathrm{e}^{-s}\right)^{2}$, which, by using for instance Tauberian theorems for Laplace transforms, leads to the logarithmic correction in time. Notice that our results are asymptotic and they do not depend on the geometric features of the billiard (radius to

cell size ratio), which would of course modify transient behaviour, as well as prefactors (with dramatic effects once this ratio tends to 0 or 1 ).

\section{Conclusions}

In this paper we have proposed a zeta function technique to compute the asymptotic behaviour of different transport moments: the essential ingredient in the analysis of weakly chaotic examples, that show deviations from the normal diffusing case, has been shown to be a proper characterization of the sequence of periodic orbits probing closer and closer dynamical features of the marginal structures. In particular the behaviour of the moments' spectrum $\nu(q)$ is governed by the power law relating such orbits' instability to their period. In many cases $\nu(q)$ is characterized by a phase transition between a normal regime and a ballistic one.

\section{Acknowledgments}

This work was partially supported by INFM PA project Weak chaos: theory and applications, and by EU contract QTRANS Network (Quantum transport on an atomic scale).

\section{References}

[1] Geisel T and Nierwetberg J 1982 Phys. Rev. Lett. 48 7; Shell M, Fraser S and Kapral R 1982 Phys. Rev. A 26504

[2] Chirikov B V 1979 Phys. Rep. 52 264; Rechester A B and White R B 1980 Phys. Rev. Lett. 44 1586

[3] Lorentz H A 1905 Proc. R. Acad. Sci. Amsterdam 7 438; Hauge E H 1974 Lect. Notes Phys 31 337 (Heidelberg: Springer); Gallavotti G 1975 Lect. Notes Phys 38236 (Heidelberg: Springer); Bunimovich L A and Sinai Ya G 1981 Commun. Math. Phys. 78479

[4] Artuso R, Aurell E and Cvitanović P 1990 Nonlinearity 3 325; Artuso R, Aurell E and Cvitanović P 1990 Nonlinearity 3 361; Cvitanović P, Artuso R, Dahlqvist P, Mainieri R, Tanner G, Vattay G, Whelan N and Wirzba A 2003 Chaos: classical and quantum (www.nbi.dk/ChaosBook/)

[5] Artuso R 1991 Phys.Lett. A 160528 
[6] Cvitanović P, Gaspard P and Schreiber T 1992 Chaos 2 85; Cvitanović P, Eckmann J-P and Gaspard P 1995 Chaos, Solitons and Fractals 6113

[7] Artuso R and Strepparava R 1997 Phys.Lett. A 236469

[8] Geisel T and Thomae S 1984 Phys. Rev. Lett. 52 1936; Geisel T, Nierwetberg J and Zacherl A 1985 Phys.Rev.Lett. 54616

[9] Benkadda S, Kassibrakis S, White R B and Zaslavsky G M 1997 Phys.Rev. E 554909

[10] Bunimovich L A 1985 Sov.Phys. JETP 62 842, Bleher P M 1992 J. Stat. Phys. 66 315; Dahlqvist P 1996 J. Stat. Phys. 84773

[11] See for instance Hoover W G 1991 Computational Statistical Mechanics (Elseview, Amsterdam)

[12] Castiglione P, Mazzino A, Muratore-Ginanneschi P and Vulpiani A 1999 Physica D 13475

[13] Artuso R and Cristadoro G 2003 Phys.Rev.Lett. 90244101

[14] Baladi V 2000 Positive transfer operators and decay of correlations (World Scientific, Singapore)

[15] Pikovsky A S 1991 Phys.Rev. A 433146

[16] Armstead D, Hunt B and Ott E 2003 Phys. Rev. E 67 021110; 2002 Phys.Rev.Lett. 89284101

[17] Klages R and Dorfman J R 1995 Phys. Rev. Lett.74 387

[18] Gallavotti G 1977, Rendiconti dell'Accademia Nazionale dei Lincei 51509

[19] Artuso R, Cvitanović P and Tanner G 2003 Cycle expansions for intermittent maps, Prog. Theor.Phys. Suppl. to appear

[20] Dahlqvist P 1999 Phys.Rev.E 60 6639; Dahlqvist P and Artuso R 1996 Phys.Lett. A 219 212; Dahlqvist P 1997 Nonlinearity 10159

[21] Prellberg T 2003 J. Phys. A 36 2455-2461

[22] Isola S 2002 Nonlinearity 15, 1521

[23] Artuso R, Casati G and Lombardi R 1993 Phys.Rev.Lett. 7162

[24] Halsey T C, Jensen M H, Kadanoff L P, Procaccia I and Shraiman B I 1986 Phys. Rev. A 33 1141; Feigenbaum M J 1987 J. Stat. Phys. 46919

[25] Artuso R, Cvitanović P and Kenny B 1989 Phys. Rev. A 39268

[26] Manneville P and Pomeau Y 1979 Phys.Lett. A 75 1; Manneville P and Pomeau Y 1980 Commun.Math.Phys. $\mathbf{7 4} 189$

[27] Gaspard P and Wang X-J 1988 Proc.Natl.Acad.Sci. U.S.A. 85 4591; Wang X-J 1989 Phys.Rev. A 406647

[28] Isola S 1995 Dynamical zeta functions for non-uniformly hyperbolic transformations Preprint mp-arc 95-142

[29] Campanino M and Isola S 1996 Forum Math. 871

[30] Artuso R and Prampolini A 1998 Phys. Lett. A 246,407 